\title{
道路橋の最大活荷重特性に関する 確率分布の解析解
}

\section{久保雅邦*・亀田弘行**}

本ノ一トは先の論文内容を補足するもので，活荷重列を受ける橋梁部材の静的な応答 を対象として, 着目点載荷と渋滞荷重列の組合せによる最大活荷重の確率特性を評価す るために，裾切りした 2 変数の指数分布のたたみ込み積分による解析解のすべてのケー スと， 3 変数の指数分布による代表的なケースの解析解を提示した.これによって，最 大活荷重特性に関する確率分布の裾部の理論的な理解が容易になった。

Keywords : probability distribution, live load, highway bridge, convolution

\section{1. まえがき}

道路橋の活荷重に対する安全性や使用性の大きさを評 価するためには，ある期間における最大活荷重の特性に ついて知ることが一つの課題になる。たとえば，主桁な どの主構造においては，渋滞時のような活荷重列による 応答の最大值が支配的な意味をもち，そのような荷重状 態を推定するとともに, 最大応答の確率分布における裾 部の特性や活荷重の実態特性との関係などを明らかにし なければならない。しかも，一般には橋梁の供用期間に 応じて，比較的長い期間の応答值が対象になるため，効 率的な解析手法が必要になる.

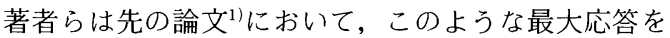
解析する方法として, 橋梁上の活荷重列を着目点載荷と それ以外の渋滞荷重列，さらには他の車線上の活荷重列 などに分離して考え，それらの荷重や荷重列による応答 の確率分布をたたみ込み積分する解析を示した。 その際, 個々の確率分布に解析可能な理論モデルを採用すれば， 最大応答の確率分布における特徴的な諸性質を理論的に 理解することができる．なお，同論文では 2 変数の指数 分布モデルによる解析解の一部のみを示した。

本ノートでは，この手法を容易に適用し得るようにす るために，2 変数の指数分布モデルによる解析解のすべ ての場合を示すとともに，3 変数の指数分布モデルによ る解析解をも提示する.

\section{2. 確率分布の解析解}

\section{（1）指数分布モデルの仮定}

橋梁上の配列が不規則な活荷重列に関して，ある着目

*正会員 工修 綜合技術コンサルタント技術開発部課長 （价33 大阪市東淀川区東中島 3-5-9）

**正会員 工博 京都大学防災研究所教授 都市施設耐震シス テム研究センター（テ611 宇治市五ヶ庄）
点上に車種 $k$ の荷重が載荷し，その前後左右に渋滞時 の活荷重列が載荷している状態を事象 $E_{k}$ として考える

(図一1). 事象 $E_{k}$ が発生しているという条件のもとで, 着目点載荷による応答値を $Y_{k}^{(1)}$, 同じ車線上の渋滞荷重 列による応答值を $Y_{k}^{(2)}$ ，および他の車線上の活荷重列に よる応答値を $Y_{k}^{(3)}$ とすれば，重ね合わせの原理からこ れらの和によって応答值 $Y_{k}$ を求めることができる.

$$
Y_{k}=Y_{k}^{(1)}+Y_{k}^{(2)}+Y_{k}^{(3)} \quad(k=1,2, \cdots) \cdots
$$

そこで， $Y_{k}^{(1)}, Y_{k}^{(2)}, Y_{k}^{(3)}$ のおのおのについて,$Y_{k}^{(1)}$ $=y_{1}, \quad Y_{k}^{(2)}=y_{2}, \quad Y_{k}^{(3)}=y_{3}$ となる確率密度関数として, それぞれ次の裙切りした指数分布 $f_{1}\left(y_{1}\right), f_{2}\left(y_{2}\right), f_{3}\left(y_{3}\right)$ を仮定する1). ただし， $a_{1}, a_{2}, a_{3}, b_{1}, b_{2}, b_{3}>0$ ，お よび $b_{0} \geqq 0$ とする。

$$
f_{1}\left(y_{1}\right)=\left\{\begin{array}{ll}
A_{1} e^{-a_{1}\left(y_{1}-b_{0}\right)} & : b_{0} \leqq y_{1} \leqq b_{1} \\
0 & : y_{1}<b_{0}, \quad b_{1}<y_{1}
\end{array}\right\}
$$

ここに, $A_{1}=\frac{a_{1}}{1-e^{-a_{1}\left(b_{1}-b_{0}\right)}}$

$$
f_{2}\left(y_{2}\right)=\left\{\begin{array}{ll}
A_{2} e^{-a_{2} y_{2}} & : 0 \leqq y_{2} \leqq b_{2} \\
0 & : y_{2}<0, \quad b_{2}<y_{2}
\end{array}\right\}
$$

ここに, $A_{2}=\frac{a_{2}}{1-e^{-a_{2} b_{2}}}$

$$
f_{3}\left(y_{3}\right)=\left\{\begin{array}{ll}
A_{3} e^{-a_{3} y_{3}} & : 0 \leqq y_{3} \leqq b_{3} \\
0 & : y_{3}<0, \quad b_{3}<y_{3}
\end{array}\right\}
$$

ここに, $A_{3}=\frac{a_{3}}{1-e^{-a_{3} b_{3}}}$

それぞれの確率密度関数を図一 2 に示す. $f_{1}\left(y_{1}\right)$ の境 界值 $b_{0}$ と $b_{1}$ は着目点載荷として考える車両重量の分布 の範囲から決まり, $f_{2}\left(y_{2}\right)$ と $f_{3}\left(y_{3}\right)$ の境界值 $b_{2}$ 之 $b_{3}$ は それぞれの活荷重列による応答値の上限値を表す.

なお，ここでの解析解は指数分布の仮定が成り立つ場 合に意味がある、したがって，このような応答值に対し て指数分布を仮定することについて議論しなければなら ないが，文献1）と2）の例題が示すように，分布の裙 


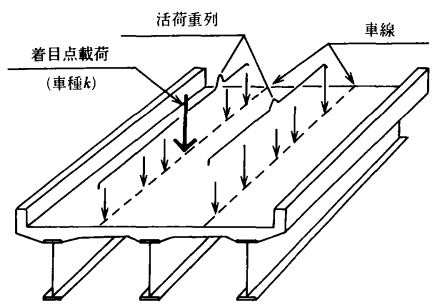

図一1 不規則な活荷重列を受ける橋梁モデルの例
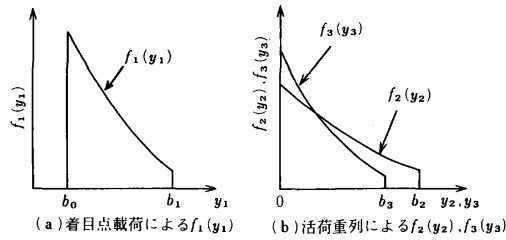

図一2 裾切りした指数分布による応答値のモデル

部に関してはおおむね妥当なものと考えられる.

\section{（2） 2 変数のたたみ込み積分}

たとえば, 1 車線上の活荷重列のみを考える場合には, $Y_{k}^{(3)}=0$ として $f_{1}\left(y_{1}\right)$ と $f_{2}\left(y_{2}\right)$ のたたみ込み積分をする ことによって， $Y_{k}=y$ よなる確率密度関数 $f_{0}(y)$ および 累積分布関数 $F_{0}(y)$ を次のように求めることができる.

$$
\begin{aligned}
& f_{0}(y)=\int_{0}^{\infty} f_{1}(y-t) f_{2}(t) d t \\
& F_{0}(y)=\int_{0}^{y} f_{0}(y) d y \cdots \cdots \cdots
\end{aligned}
$$

分布のパラメーター $a_{1}, a_{2}$ および $b_{0}, b_{1}, b_{2}$ の大小 関係に応じて, 式 (5) と式 (6) の解析解を以下に示 す.ただし，表記上，次の記号を用いる.

$$
\left.\begin{array}{l}
B_{0}=\frac{A_{1} A_{2}}{\left(a_{1}-a_{2}\right)^{2}}, \quad B_{1}=\frac{A_{1} A_{2}}{\left(a_{1}\right)^{2}} \\
E_{1}=e^{-a_{1}\left(b_{1}-b_{0}\right)}, \quad E_{2}=e^{-a_{2} b_{2}} \\
\beta_{1}=\frac{a_{2}-a_{1}}{a_{1}}, \quad \beta_{2}=\frac{a_{1}-a_{2}}{a_{2}}
\end{array}\right\}
$$

\section{a) $a_{1} \neq a_{2}, \quad\left(b_{1}-b_{0}\right) \geqq b_{2}$ の場合}

以下を式 $(8)$ とする

1) $y<b_{0}: f_{0}(y)=0, \quad F_{0}(y)=0$

2) $b_{0} \leqq y<b_{0}+b_{2}: f_{0}(y)=B_{0}\left[\left(a_{2}-a_{1}\right) e^{-a_{1}\left(y-b_{0}\right)}+\left(a_{1}-a_{2}\right)\right.$ $\left.e^{-a_{2}\left(y-b_{0}\right)}\right], \quad F_{0}(y)=B_{0}\left\{\beta_{1}\left[1-e^{-a_{1}\left(y-b_{0}\right)}\right]+\beta_{2}[1-\right.$ $\left.\left.e^{-a_{2}(y-b 0)}\right]\right\}$

3) $b_{0}+b_{2} \leqq y<b_{1}: f_{0}(y)=B_{0}\left[\left(a_{2}-a_{1}\right) e^{-a_{1}\left(y-b_{0}\right)}+\left(a_{1}-a_{2}\right)\right.$ $\left.E_{2} e^{-a_{1}\left(y-b_{0}-b_{2}\right)}\right], \quad F_{0}(y)=B_{0}\left\{\beta_{1}\left[1-E_{2}-e^{-a_{1}\left(y-b_{0}\right)}+\right.\right.$ $\left.\left.E_{2} e^{-a_{1}\left(y-b_{0}-b_{2}\right)}\right]+\beta_{2}\left(1-E_{2}\right)\right\}$

4) $b_{1} \leqq y<b_{1}+b_{2}: f_{0}(y)=B_{0}\left[\left(a_{2}-a_{1}\right) E_{1} e^{-a_{2}\left(y-b_{1}\right)}+\left(a_{1}-\right.\right.$ $\left.\left.a_{2}\right) E_{2} e^{-a_{1}\left(y-b_{0}-b_{2}\right)}\right], \quad F_{0}(y)=B_{0}\left\{\beta_{1}\left[1-E_{1}-E_{2}+\right.\right.$
$\left.\left.E_{2} e^{-a_{1}\left(y-b_{0}-b_{2}\right)}\right]+\beta_{2}\left[1-E_{1}-E_{2}+E_{1} e^{-a_{2}\left(y-b_{1}\right)}\right]\right\}$

5) $b_{1}+b_{2} \leqq y: f_{0}(y)=0, F_{0}(y)=1$

b) $a_{1} \neq a_{2}, \quad b_{2}>\left(b_{1}-b_{0}\right)$ の場合

………………....以下式( 9 ) とする

1) $y<b_{0}: f_{0}(y)=0, \quad F_{0}(y)=0$

2) $b_{0} \leqq y<b_{1}: f_{0}(y), F_{0}(y)$ は式 $(8)$ の 2$)$ に同じ

3) $b_{1} \leqq y<b_{0}+b_{2}: f_{0}(y)=B_{0}\left[\left(a_{2}-a_{1}\right) E_{1} e^{-a_{2}\left(y-b_{1}\right)}+\left(a_{1}-\right.\right.$ $\left.\left.a_{2}\right) e^{-a_{2}\left(y-b_{0}\right)}\right], \quad F_{0}(y)=B_{0}\left\{\beta_{1}\left(1-E_{1}\right)+\beta_{2}\left[1-E_{1}+\right.\right.$ $\left.\left.E_{1} e^{-a_{2}\left(y-b_{1}\right)}-e^{-a_{2}\left(y-b_{0}\right)}\right]\right\}$

4) $b_{0}+b_{2} \leqq y<b_{1}+b_{2}: f_{0}(y), F_{0}(y)$ は式（8）の 4$)$ に 同じ

5) $b_{1}+b_{2} \leqq y: f_{0}(y)=0, F_{0}(y)=1$

c ) $a_{1}=a_{2}, \quad\left(b_{1}-b_{0}\right) \geqq b_{2}$ の場合 以下を式(10)とする

1) $y<b_{0}: f_{0}(y)=0, \quad F_{0}(y)=0$

2) $b_{0} \leqq y<b_{0}+b_{2}: f_{0}(y)=A_{1} A_{2}\left(y-b_{0}\right) e^{-a_{1}\left(y-b_{0}\right)}, F_{0}(y)=$ $B_{1}\left\{1-\left[1+a_{1}\left(y-b_{0}\right)\right] e^{-a_{1}\left(y-b_{0}\right)}\right\}$

3) $b_{0}+b_{2} \leqq y<b_{1}: f_{0}(y)=A_{1} A_{2} b_{2} e^{-a_{1}\left(y-b_{0}\right)}, \quad F_{0}(y)=B_{1}[1$ $\left.-E_{2}-a_{1} b_{2} e^{-a_{1}\left(y-b_{0}\right)}\right]$

4) $b_{1} \leqq y<b_{1}+b_{2}: f_{0}(y)=A_{1} A_{2}\left(b_{1}+b_{2}-y\right) e^{-a_{1}\left(y-b_{0}\right)}, F_{0}(y)$ $=B_{1}\left\{1-E_{1}-E_{2}+\left[1-a_{1}\left(b_{1}+b_{2}-y\right)\right] e^{-a_{1}\left(y-b_{0}\right)}\right\}$

5) $b_{1}+b_{2} \leqq y: f_{0}(y)=0, F_{0}(y)=1$

d) $a_{1}=a_{2}, b_{2}>\left(b_{1}-b_{0}\right)$ の場合 …………………以下を式(11) とする

1) $y<b_{0}: f_{0}(y)=0, \quad F_{0}(y)=0$

2) $b_{0} \leqq y<b_{1}: f_{0}(y), F_{0}(y)$ は式 (10) の 2$)$ に同じ

3) $b_{1} \leqq y<b_{0}+b_{2}: f_{0}(y)=A_{1} A_{2}\left(b_{1}-b_{0}\right) e^{-a_{1}\left(y-b_{0}\right)}, F_{0}(y)=$ $B_{1}\left[1-E_{1}-a_{1}\left(b_{1}-b_{0}\right) e^{-a_{1}\left(y-b_{0}\right)}\right]$

4) $b_{0}+b_{2} \leqq y<b_{1}+b_{2}: f_{0}(y), F_{0}(y)$ は式 (10) の 4$)$ に 同じ

5) $b_{1}+b_{2} \leqq y: f_{0}(y)=0, F_{0}(y)=1$

（３） 3 変数のたたみ込み積分

式（1）に基づいて $Y_{k}^{(1)}, Y_{k}^{(2)}, Y_{k}^{(3)}$ を考える場合に は， 3 変数のたたみ込み積分によって， $Y_{k}=y$ となる確 率密度関数 $g_{0}(y)$ および累積分布関数 $G_{0}(y)$ を次のよ うに求めることができる.

$$
\begin{aligned}
& g_{0}(y)=\int_{0}^{\infty} \int_{0}^{\infty} f_{1}(y-s) f_{2}(s-t) f_{3}(t) d t d s \\
& G_{0}(y)=\int_{0}^{y} g_{0}(y) d y \ldots \ldots \ldots \ldots \ldots \ldots \ldots \ldots \ldots \ldots \ldots \ldots \ldots \ldots \ldots \ldots
\end{aligned}
$$

分布のパラメーター $a_{1}, a_{2}, a_{3}$ の等号亡不等号の関 係に応じて 5 種類，および $b_{0}, b_{1}, b_{2}, b_{3}$ の大小関係に 応じて 6 種類, したがって式 (12) と式 (13) 加ら厳密 には $5 \times 6=30$ 通りの解析解が得られるが, 紙面の都合 
により最も生じやすい 4 通りの結果を以下に示す。ただ し，表記上，式（7）に加えて次の記号を用いる.

$$
\left.\begin{array}{l}
C_{0}=-\frac{A_{1} A_{2} A_{3}}{\left(a_{1}-a_{2}\right)\left(a_{2}-a_{3}\right)\left(a_{3}-a_{1}\right)} \\
E_{3}=e^{-a_{3} b_{3}} \\
r_{1}=\frac{a_{2}-a_{3}}{a_{1}}, \quad r_{2}=\frac{a_{3}-a_{1}}{a_{2}}, \quad \gamma_{3}=\frac{a_{1}-a_{2}}{a_{3}}
\end{array}\right\}
$$

\section{a) $a_{1} \neq a_{2} \neq a_{3}, \quad\left(b_{2}+b_{3}\right) \geqq\left(b_{1}-b_{0}\right) \geqq b_{2} \geqq b_{3}$ の場合} .以下を式(15)とする

1) $y<b_{0}: g_{0}(y)=0, \quad G_{0}(y)=0$

2) $b_{0} \leqq y<b_{0}+b_{3}: g_{0}(y)=C_{0}\left[\left(a_{2}-a_{3}\right) e^{-a_{1}\left(y-b_{0}\right)}+\left(a_{3}-a_{1}\right)\right.$ $\left.e^{-a_{2}\left(y-b_{0}\right)}+\left(a_{1}-a_{2}\right) e^{-a_{3}\left(y-b_{0}\right)}\right], \quad G_{0}(y)=C_{0}\left\{\gamma_{1}[1-\right.$ $\left.\left.e^{-a_{1}\left(y-b_{0}\right)}\right]+\gamma_{2}\left[1-e^{-a_{2}\left(y-b_{0}\right)}\right]+\gamma_{3}\left[1-e^{-a_{3}\left(y-b_{0}\right)}\right]\right\}$

3) $b_{0}+b_{3} \leqq y<b_{0}+b_{2}: g_{0}(y)=C_{0}\left\{\left(a_{2}-a_{3}\right)\left[e^{-a_{1}\left(y-b_{0}\right)}-\right.\right.$ $\left.\left.E_{3} e^{-a_{1}\left(y-b_{0}-b_{3}\right)}\right]+\left(a_{3}-a_{1}\right)\left[e^{-a_{2}\left(y-b_{0}\right)}-E_{3} e^{-a_{2}\left(y-b_{0}-b_{3}\right)}\right]\right\}$, $G_{0}(y)=C_{0}\left\{\gamma_{1}\left[1-E_{3}-e^{-a_{1}\left(y-b_{0}\right)}+E_{3} e^{-a_{1}\left(y-b_{0}-b_{3}\right)}\right]+\gamma_{2}[1-\right.$ $\left.\left.E_{3}-e^{-a_{2}\left(y-b_{0}\right)}+E_{3} e^{-a_{2}\left(y-b_{0}-b_{3}\right)}\right]+\gamma_{3}\left(1-E_{3}\right)\right\}$

4) $b_{0}+b_{2} \leqq y<b_{1}: g_{0}(y)=C_{0}\left\{\left(a_{2}-a_{3}\right)\left[e^{-a_{1}\left(y-b_{0}\right)}-\right.\right.$ $\left.E_{2} e^{-a_{1}\left(y-b_{0}-b_{2}\right)}-E_{3} e^{-a_{1}\left(y-b_{0}-b_{3}\right)}\right]-\left(a_{3}-a_{1}\right) E_{3} e^{-a_{2}\left(y-b_{0}-b_{3}\right)}-$ $\left.\left(a_{1}-a_{2}\right) E_{2} e^{-a_{3}\left(y-b_{0}-b_{2}\right)}\right\}, \quad G_{0}(y)=C_{0}\left\{r_{1}\left[1-E_{2}-E_{3}-\right.\right.$ $\left.e^{-a_{1}\left(y-b_{0}\right)}+E_{2} e^{-a_{1}\left(y-b_{0}-b_{2}\right)}+E_{3} e^{-a_{1}\left(y-b_{0}-b_{3}\right)}\right]+\gamma_{2}\left[1-E_{2}-E_{3}\right.$ $\left.\left.+E_{3} e^{-a_{2}\left(y-b_{0}-b_{3}\right)}\right]+\gamma_{3}\left[1-E_{2}-E_{3}+E_{2} e^{-a_{3}\left(y-b_{0}-b_{2}\right)}\right]\right\}$

5) $b_{1} \leqq y<b_{0}+b_{2}+b_{3}: g_{0}(y)=C_{0}\left\{-\left(a_{2}-a_{3}\right)\right.$ $\left[E_{2} e^{-a_{1}\left(y-b_{0}-b_{2}\right)}+E_{3} e^{-a_{1}\left(y-b_{0}-b_{3}\right)}\right]-\left(a_{3}-a_{1}\right)\left[E_{1} e^{-a_{2}\left(y-b_{1}\right)}+\right.$ $\left.\left.E_{3} e^{-a_{2}\left(y-b_{0}-b_{3}\right)}\right]-\left(a_{1}-a_{2}\right)\left[E_{1} e^{-a_{3}\left(y-b_{1}\right)}+E_{2} e^{-a_{3}\left(y-b_{0}-b_{2}\right)}\right]\right\}$, $G_{0}(y)=C_{0}\left\{\gamma_{1}\left[1-E_{1}-E_{2}-E_{3}+E_{2} e^{-a_{1}\left(y-b_{0}-b_{2}\right)}+\right.\right.$ $\left.E_{3} e^{-a_{1}\left(y-b_{0}-b_{3}\right)}\right]+\gamma_{2}\left[1-E_{1}-E_{2}-E_{3}+E_{1} e^{-a_{2}\left(y-b_{1}\right)}+\right.$ $\left.E_{3} e^{-a_{2}\left(y-b_{0}-b_{3}\right)}\right]+\gamma_{3}\left[1-E_{1}-E_{2}-E_{3}+E_{1} e^{-a_{3}\left(y-b_{1}\right)}+\right.$ $\left.\left.E_{2} e^{-a_{3}\left(y-b_{0}-b_{2}\right)}\right]\right\}$

6) $b_{0}+b_{2}+b_{3} \leqq y<b_{1}+b_{3}: g_{0}(y)=C_{0}\left\{-\left(a_{2}-a_{3}\right)\right.$ $\left[E_{2} e^{-a_{1}\left(y-b_{0}-b_{2}\right)}+E_{3} e^{-a_{1}\left(y-b_{0}-b_{3}\right)}-E_{2} E_{3} e^{-a_{1}\left(y-b_{0}-b_{2}-b_{3}\right)}\right]-\left(a_{3}\right.$ $\left.\left.-a_{1}\right) E_{1} e^{-a_{2}\left(y-b_{1}\right)}-\left(a_{1}-a_{2}\right) E_{1} e^{-a_{3}\left(y-b_{1}\right)}\right\}, \quad G_{0}(y)=C_{0}\left\{\gamma_{1}[1\right.$ $-E_{1}-E_{2}-E_{3}+E_{2} E_{3}+E_{2} e^{-a_{1}\left(y-b_{0}-b_{2}\right)}+E_{3} e^{-a_{1}\left(y-b_{0}-b_{3}\right)}-$ $\left.E_{2} E_{3} e^{-a_{1}\left(y-b_{0}-b_{2}-b_{3}\right)}\right]+\gamma_{2}\left[1-E_{1}-E_{2}-E_{3}+E_{2} E_{3}+\right.$ $\left.\left.E_{1} e^{-a_{2}\left(y-b_{1}\right)}\right]+\gamma_{3}\left[1-E_{1}-E_{2}-E_{3}+E_{2} E_{3}+E_{1} e^{-a_{3}\left(y-b_{1}\right)}\right]\right\}$

7) $b_{1}+b_{3} \leqq y<b_{1}+b_{2}: g_{0}(y)=C_{0}\left\{-\left(a_{2}-a_{3}\right)\right.$ $\left[E_{2} e^{-a_{1}\left(y-b_{0}-b_{2}\right)}-E_{2} E_{3} e^{-a_{1}\left(y-b_{0}-b_{2}-b_{3}\right)}\right]-\left(a_{3}-a_{1}\right)\left[E_{1} e^{-a_{2}\left(y-b_{1}\right)}\right.$ $\left.\left.-E_{1} E_{3} e^{-a_{2}\left(y-b_{1}-b_{3}\right)}\right]\right\}, \quad G_{0}(y)=C_{0}\left\{\gamma_{1}\left[1-E_{1}-E_{2}-E_{3}+\right.\right.$ $\left.E_{1} E_{3}+E_{2} E_{3}+E_{2} e^{-a_{1}\left(y-b_{0}-b_{2}\right)}-E_{2} E_{3} e^{-a_{1}\left(y-b_{0}-b_{2}-b_{3}\right)}\right]+\gamma_{2}[1-$ $E_{1}-E_{2}-E_{3}+E_{1} E_{3}+E_{2} E_{3}+E_{1} e^{-a_{2}\left(y-b_{1}\right)}-$ $\left.\left.E_{1} E_{3} e^{-a_{2}\left(y-b_{1}-b_{3}\right)}\right]+\gamma_{3}\left(1-E_{1}-E_{2}-E_{3}+E_{1} E_{3}+E_{2} E_{3}\right)\right\}$
8) $b_{1}+b_{2} \leqq y<b_{1}+b_{2}+b_{3}: g_{0}(y)=C_{0}\left\{\left(a_{2}-a_{3}\right)\right.$ $E_{2} E_{3} e^{-a_{1}\left(y-b_{0}-b_{2}-b_{3}\right)}+\left(a_{3}-a_{1}\right) E_{1} E_{3} e^{-a_{2}\left(y-b_{1}-b_{3}\right)}+\left(a_{1}-a_{2}\right)$ $\left.E_{1} E_{2} e^{-a_{3}\left(y-b_{1}-b_{2}\right)}\right\}, G_{0}(y)=\left\{C_{0}\left\{\gamma_{1}\left[1-E_{1}-E_{2}-E_{3}+E_{1} E_{2}\right.\right.\right.$ $\left.+E_{2} E_{3}+E_{1} E_{3}-E_{2} E_{3} e^{-a_{1}\left(y-b_{0}-b_{2}-b_{3}\right)}\right]+\gamma_{2}\left[1-E_{1}-E_{2}-E_{3}\right.$ $\left.+E_{1} E_{2}+E_{2} E_{3}+E_{1} E_{3}-E_{1} E_{3} e^{-a_{2}\left(y-b_{1}-b_{3}\right)}\right]+\gamma_{3}\left[1-E_{1}-E_{2}\right.$ $\left.\left.-E_{3}+E_{1} E_{2}+E_{2} E_{3}+E_{1} E_{3}-E_{1} E_{2} e^{-a_{3}\left(y-b_{1}-b_{2}\right)}\right]\right\}$

9) $b_{1}+b_{2}+b_{3} \leqq y: g_{0}(y)=0, \quad G_{0}(y)=1$

b) $a_{1} \neq a_{2} \neq a_{3}, \quad\left(b_{1}-b_{0}\right)>\left(b_{2}+b_{3}\right)>b_{2} \geqq b_{3}$ の場合 以下を式(16)とする

1) $y<b_{0}: g_{0}(y)=0, G_{0}(y)=0$

2) $b_{0} \leqq y<b_{0}+b_{3}: g_{0}(y), G_{0}(y)$ は式(15)の 2$)$ に同じ

3) $b_{0}+b_{3} \leqq y<b_{0}+b_{2}: g_{0}(y), G_{0}(y)$ は式 (15) の 3 ) に同じ

4) $b_{0}+b_{2} \leqq y<b_{0}+b_{2}+b_{3}: g_{0}(y), G_{0}(y)$ は式 (15) の 4 ) に同じ

5) $b_{0}+b_{2}+b_{3} \leqq y<b_{1}: g_{0}(y)=C_{0}\left\{\left(a_{2}-a_{3}\right)\left[e^{-a_{1}\left(y-b_{0}\right)}-\right.\right.$ $\left.\left.E_{2} e^{-a_{1}\left(y-b_{0}-b_{2}\right)}-E_{3} e^{-a_{1}\left(y-b_{0}-b_{3}\right)}+E_{2} E_{3} e^{-a_{1}\left(y-b_{0}-b_{2}-b_{3}\right)}\right]\right\}$, $G_{0}(y)=C_{0}\left\{\gamma_{1}\left[1-E_{2}-E_{3}+E_{2} E_{3}-e^{-a_{1}\left(y-b_{0}\right)}+\right.\right.$ $\left.E_{2} e^{-a_{1}\left(y-b_{0}-b_{2}\right)}+E_{3} e^{-a_{1}\left(y-b_{0}-b_{3}\right)}-E_{2} E_{3} e^{-a_{1}\left(y-b_{0}-b_{2}-b_{3}\right)}\right]+\gamma_{2}[1$ $\left.\left.-E_{2}-E_{3}+E_{2} E_{3}\right]+\gamma_{3}\left[1-E_{2}-E_{3}+E_{2} E_{3}\right]\right\}$

6) $b_{1} \leqq y<b_{1}+b_{3}: g_{0}(y) ， G_{0}(y)$ は式(15)の 6$)$ に同じ

7) $b_{1}+b_{3} \leqq y<b_{1}+b_{2}: g_{0}(y), G_{0}(y)$ は式 (15) の 7 ) に同じ

8) $b_{1}+b_{2} \leqq y<b_{1}+b_{2}+b_{3}: g_{0}(y), G_{0}(y)$ は式 (15) の 8 ) に同じ

9) $b_{1}+b_{2}+b_{3} \leqq y: g_{0}(y)=0, \quad G_{0}(y)=1$

c) $a_{1} \neq a_{2} \neq a_{3}, \quad\left(b_{1}-b_{0}\right)+b_{3} \geqq b_{2}>\left(b_{1}-b_{0}\right) \geqq b_{3}$ の場 合…………………....以下を式(17) とする

1) $y<b_{0}: g_{0}(y)=0, \quad G_{0}(y)=0$

2) $b_{0} \leqq y<b_{0}+b_{3}: g_{0}(y), G_{0}(y)$ は式(15)の 2$)$ に同じ

3) $b_{0}+b_{3} \leqq y<b_{1}: g_{0}(y), G_{0}(y)$ は式(15)の 3$)$ に同じ

4) $b_{1} \leqq y<b_{0}+b_{2}: g_{0}(y)=C_{0}\left\{-\left(a_{2}-a_{3}\right) E_{3} e^{-a_{1}\left(y-b_{0}-b_{3}\right)}+\right.$ $\left(a_{3}-a_{1}\right)\left[e^{-a_{2}\left(y-b_{0}\right)}-E_{1} e^{-a_{2}\left(y-b_{1}\right)}-E_{3} e^{-a_{2}\left(y-b_{0}-b_{3}\right)}\right]-\left(a_{1}-a_{2}\right)$ $\left.E_{1} e^{-a_{3}\left(y-b_{1}\right)}\right\}, \quad G_{0}(y)=C_{0}\left\{r_{1}\left[1-E_{1}-E_{3}+E_{3} e^{-a_{1}\left(y-b_{0}-b_{3}\right)}\right]\right.$ $+\gamma_{2}\left[1-E_{1}-E_{3}-e^{-a_{2}\left(y-b_{0}\right)}+E_{1} e^{-a_{2}\left(y-b_{1}\right)}+E_{3} e^{-a_{2}\left(y-b_{0}-b_{3}\right)}\right]$ $\left.+\gamma_{3}\left[1-E_{1}-E_{3}+E_{1} e^{-a_{3}\left(y-b_{1}\right)}\right]\right\}$

5) $b_{0}+b_{2} \leqq y<b_{1}+b_{3}: g_{0}(y), G_{0}(y)$ は式（15）の 5 ) に同じ

6) $b_{1}+b_{3} \leqq y<b_{0}+b_{2}+b_{3}: g_{0}(y)=C_{0}\left\{-\left(a_{2}-a_{3}\right)\right.$ $E_{2} e^{-a_{1}\left(y-b_{0}-b_{2}\right)}-\left(a_{3}-a_{1}\right)\left[E_{1} e^{-a_{2}\left(y-b_{1}\right)}+E_{3} e^{-a_{2}\left(y-b_{0}-b_{3}\right)}-\right.$ $\left.\left.E_{1} E_{3} e^{-a_{2}\left(y-b_{1}-b_{3}\right)}\right]-\left(a_{1}-a_{2}\right) E_{2} e^{-a_{3}\left(y-b_{0}-b_{2}\right)}\right\}, \quad G_{0}(y)=C_{0}$ $\left\{\gamma_{1}\left[1-E_{1}-E_{2}-E_{3}+E_{1} E_{3}+E_{2} e^{-a_{1}\left(y-b_{0}-b_{2}\right)}\right]+\gamma_{2}\left[1-E_{1}-\right.\right.$ 
$E_{2}-E_{3}+E_{1} E_{3}+E_{1} e^{-a_{2}\left(y-b_{1}\right)}+E_{3} e^{-a_{2}\left(y-b_{0}-b_{3}\right)}-$ $\left.E_{1} E_{3} e^{-a_{2}\left(y-b_{1}-b_{3}\right)}\right]+\gamma_{3}\left[1-E_{1}-E_{2}-E_{3}+E_{1} E_{3}+\right.$ $\left.\left.E_{2} e^{-a_{3}\left(y-b_{0}-b_{2}\right)}\right]\right\}$

7) $b_{0}+b_{2}+b_{3} \leqq y<b_{1}+b_{2}: g_{0}(y), G_{0}(y)$ は式 (15) の 7$)$ に同じ

8) $b_{1}+b_{2} \leqq y<b_{1}+b_{2}+b_{3}: g_{0}(y), G_{0}(y)$ は式 (15) の 8$)$ に同じ

9) $b_{1}+b_{2}+b_{3} \leqq y: g_{0}(y)=0, G_{0}(y)=1$

d) $a_{1} \neq a_{2} \neq a_{3}, \quad b_{2}>\left(b_{1}-b_{0}\right)+b_{3}>\left(b_{1}-b_{0}\right) \geqq b_{3}$ の場 合………………….以下を式 $(18)$ とする

1) $y<b_{0}: g_{0}(y)=0, \quad G_{0}(y)=0$

2) $b_{0} \leqq y<b_{0}+b_{3}: g_{0}(y), G_{0}(y)$ は式(15)の 2$)$ に同じ

3) $b_{0}+b_{3} \leqq y<b_{1}: g_{0}(y), G_{0}(y)$ は式(15)の 3$)$ に同じ

4) $b_{1} \leqq y<b_{1}+b_{3}: g_{0}(y), G_{0}(y)$ は式(17)の 4$)$ に同じ

5) $b_{1}+b_{3} \leqq y<b_{0}+b_{2}: g_{0}(y)=C_{0}\left\{\left(a_{3}-a_{1}\right)\left[e^{-a_{2}\left(y-b_{0}\right)}-\right.\right.$ $\left.\left.E_{1} e^{-a_{2}\left(y-b_{1}\right)}-E_{3} e^{-a_{2}\left(y-b_{0}-b_{3}\right)}+E_{1} E_{3} e^{-a_{2}\left(y-b_{1}-b_{3}\right)}\right]\right\}, \quad G_{0}(y)=$ $C_{0}\left\{\gamma_{1}\left[1-E_{1}-E_{3}+E_{1} E_{3}\right]+\gamma_{2}\left[1-E_{1}-E_{3}+E_{1} E_{3}+\right.\right.$ $\left.E_{1} e^{-a_{2}\left(y-b_{1}\right)}-e^{-a_{2}\left(y-b_{0}\right)}+E_{3} e^{-a_{2}\left(y-b_{0}-b_{3}\right)}-E_{1} E_{3} e^{-a_{2}\left(y-b_{1}-b_{3}\right)}\right]$ $\left.+\gamma_{3}\left[1-E_{1}-E_{3}+E_{1} E_{3}\right]\right\}$

6) $b_{0}+b_{2} \leqq y<b_{0}+b_{2}+b_{3}: g_{0}(y), G_{0}(y)$ は式 (17)の 6$)$ に同じ

7) $b_{0}+b_{2}+b_{3} \leqq y<b_{1}+b_{2}: g_{0}(y), G_{0}(y)$ は式 (15)の 7 ) に同じ

8) $b_{1}+b_{2} \leqq y<b_{1}+b_{2}+b_{3}: g_{0}(y), G_{0}(y)$ は式 (15) の 8 ) に同じ

9) $b_{1}+b_{2}+b_{3} \leqq y: g_{0}(y)=0, G_{0}(y)=1$

\section{3. 計 算 例}

2変数の場合の $f_{0}(y)$ と $F_{0}(y)$, および 3 変数の場合 の $g_{0}(y)$ と $G_{0}(y)$ に関する計算例を図一 3 に示す。た だし, 分布のパラメーターの值を図中に示すが, 応答值 $y$ は文献 1 ) や 2 ）の例題と同じく, 設計荷重で正規化 した值を表すものとする. $f_{0}(y)$ と $F_{0}(y)$ についてはケ一 ス b) の場合, $g_{0}(y)$ と $G_{0}(y)$ についてはケース c) の 場合をそれぞれ表す。

この結果から，たとえば極值 I 型確率紙上に表示した

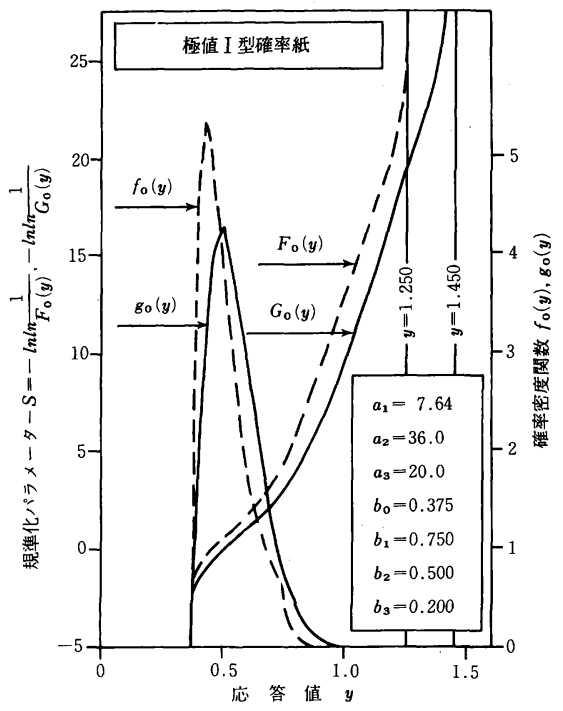

図一3 計算例

累積分布関数が，応答值 $y$ の分布範囲に応じて上側に漸 近する傾向をみることができる．また， $f_{0}(y), F_{0}(y)$ 亡 $g_{0}(y), G_{0}(y)$ のそれぞれの差から, $f_{3}\left(y_{3}\right)$ を組み合 わせた影響を定量的に把握することができる.このよう に, 確率分布の解析解を利用すれば，最大応答の確率分 布における諸性質を理論的に理解することができる．な お，本論文では 3 変数のたたみ込み積分による解析解の 代表例のみを示したが，そのすべてを誘導することがで き, コンピュータプログラム化してある. さらに, 上記 の方法を拡張することによって，4 変数以上のたたみ込 み積分にも適用することができる.

\section{参 考 文 献}

1）久保雅邦・亀田弘行 : 着目点載荷と渋滞荷重列の組合せ による道路橋の最大活荷重特性の解析, 土木学会論文集, 第 398 号 / I - 10, 1988 年 10 月.

2) Kameda, H. and Kubo, M. : Lifetime-Maximum Load Effect for Highway Bridges Based on Stochastic Combination of Typical Traffic Loadings, Proc. of ICOSSAR'89, San Francisco, Aug. 1989.

(1990.10.8 受付)

\section{CLOSED-FORM SOLUTION FOR THE PROBABILITY DISTRIBUTION OF LIFETIME MAXIMUM LIVE LOAD FOR HIGHWAY BRIDGES}

\section{Masakuni KUBO and Hiroyuki KAMEDA}

A set of closed-form solution for the probability distribution of maximum live load for highway bridges are presented by using the method developed in Refs. 1)-2). Whereas Ref. 1) contained only a part of analytical results of a single-fold convolution, their complete set is presented herein. In addition, the results for two-fold convolution are also presented herein. This will enable one to make wider application of the method. 